\title{
Reading others' minds by measuring their brains: Fascinating and challenging for science, but ready for use in court?
}

Kessler, K. \& Muckli, L.

As senior researchers in psychology and experts in functional magnetic resonance imaging (fMRI: LM) and Magneto-/Encephalography (MEG/EEG: KK), respectively, we ask the question from a scientific point of view whether the existing methods and findings in brain imaging (fMRI, EEG/MEG) could contribute to decisions in court at this point in time. Firstly, we point out the relative nature of scientific findings and the present lack of an informed dissemination of results into society. Secondly, we summarise what seems to be scientifically possible at the moment and what challenges future research must address before neuroimaging could be used in court.

Research outcomes essentially depend on the questions we ask - i.e., the theory behind the research (Popper, 1934) - and how we try to answer them - i.e., the methods of measurement (e.g., brain imaging) and analysis (e.g., statistics). Thomas Kuhn (1967) further pointed out that theories are embedded within schools of thought or paradigms, where the different schools are in competition with respect to the "scientific truth" - and as a more recent development - also with respect to research funding (Fig. 1). The arising problems are three-fold.

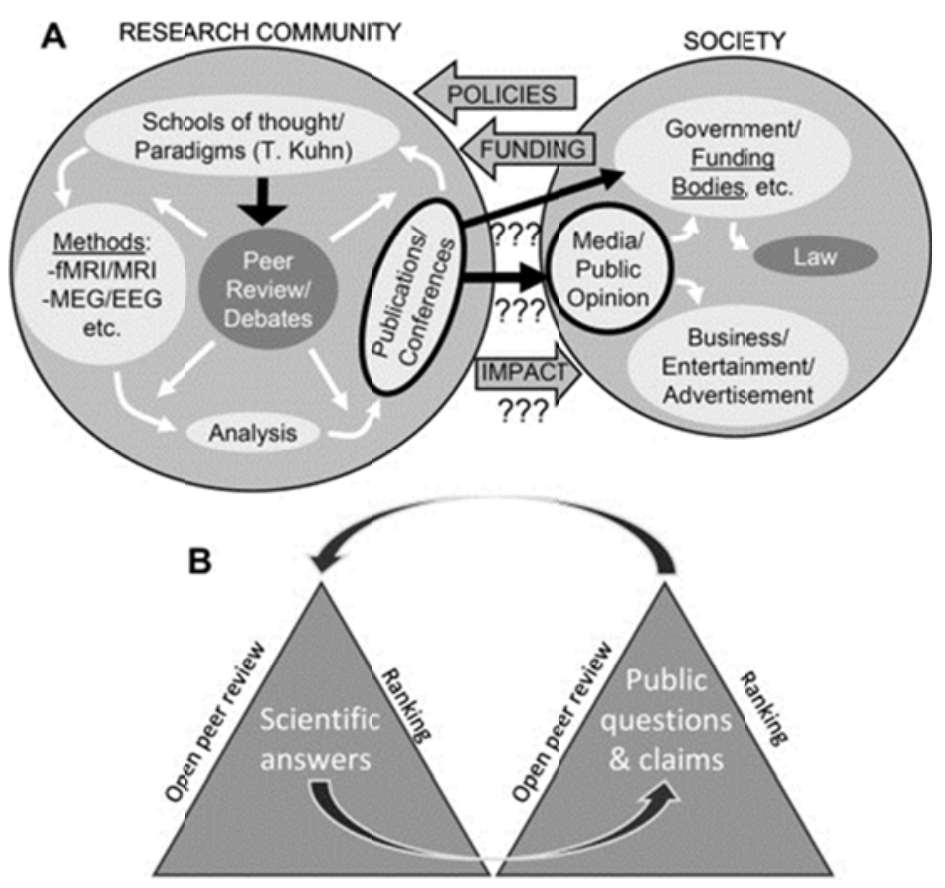

Fig. 1. - Panel A: Science and Society. The left sphere contains a schematic of the stages and regulatory mechanisms that are part of generating scientific outcomes, while the right sphere shows a simplistic schema of society and its bodies that are most strongly influenced by science. Society regulates research in return by means of policies and selective funding. The question-marks between the two spheres indicate that the dissemination of knowledge from research into society is not well enough defined and developed at the moment for ensuring an informed transfer that is not biased by sensationalist media or by the interests of the scientists themselves. Panel B: Review and ranking process proposed by Kriegeskorte et al. (in prep.) for establishing the most relevant questions and the most relevant answers from the scientific community. Public questions are ranked by the public in a peer-review and peer-ranking process. Scientists address the most important questions first (at the top of the right triangle), by proposing answers that are also subject to a peer-review and peer-ranking process conducted by the scientific community. This "two-column" procedure is suggested to replace the current question-marks in Panel A. Further explanations are provided in the text. 
Firstly, the regulatory mechanism for publishing research results, namely the process of "peer-review" cannot be regarded as fully impartial. Researchers in their role as peer reviewers are implicitly biased towards a specific school of thought (Fig. 1). Direct competition for funding may further increase the negative bias towards competitors. At the same time, however, there are no viable alternatives; thus, improving the peer-review process by ensuring the highest possible standards is the only option. Inspiring new approaches are being developed (e.g., Deca and Kriegeskorte, 2010) and newly established online journals, like the "PLoS" and "Frontiers" families, offer a novel open peer-review process, where the quality of a paper is established by post-publication review that results in a ranking based on the scientific community as a whole. Hence, while peer-review is fallible, it is the only option for the scientific community and essential improvements are possible and being explored.

Secondly, new insights may become available that question results which have been already published. Good examples are some of the recent debates in the brain imaging community:

1) Yuval-Greenberg et al. (2008) have pointed out that very subtle movements of the eyes (i.e., microsaccades) could explain some of the published findings on oscillatory gamma effects. Gamma oscillations are assumed to be essential for processes of visual integration and some of the crucial supporting findings had to be re-examined. One instance of this debate was hosted at the Centre for Cognitive Neuroimaging (CCNi) at the University of Glasgow ('CCNi events' at http://www.ccni.gla.ac.uk).

2) Sirotin and Das (2009) reported modulations of regional brain blood circulation (e.g., fMRI-signal) in the absence of electrical brain activation, which forced the scientific community to re-examine some of the simplistic interpretations of the BOLD signal (basis of $\mathrm{fMRI}$ ) in the literature. Again the CCNi hosted an instance of this debate ('CCNi events' at http://www.ccni.gla.ac.uk).

3) Kriegeskorte et al. (2009) pointed out the danger of pre-selecting brain imaging data in terms of socalled "double dipping" as this biases the data towards a particular outcome. Some findings had to be re-considered when more rigorous standards of data analysis where applied. In each of these debates one side argues that a significant body of published results have been substantially misinterpreted. These representative examples emphasise the relative and sometimes temporary nature of all knowledge in experimental sciences.

Finally, if peer-review is fallible and the scientific "truth" uncertain, then which scientific findings should be disseminated to society and how could further biasing by sensationalist media be avoided? Press releases and news coverage are much less stringently monitored than scientific publications and can bias scientific outcomes and boost their apparent importance for the broader society. Findings with limited relevance to society are sometimes exaggerated in the press as important break-throughs, above and beyond of what is reported in the actual scientific paper. We therefore propose to apply an improved process of peer-review (as outlined above) to the interface between science and society (Fig. 1, Panel B). Urgent questions to the scientific community could be posted by the public and subsequently rated and ranked by public- and nonprofit organisations. Questions of broad interest or high relevance reach the top of the ranking. Scientists would then suggest and comment on the most relevant findings regarding these questions, while the whole process is monitored by the scientific community in open peer-review, ensuring transparency, if not 
fairness. Originally, these ideas have been proposed by Kriegeskorte et al. (personal communication) and are being prepared for publication in more detail.

For now let us assume that all the problems of selecting the most reliable findings for dissemination into society have been resolved. What would be required of a method for its use in court (Table 1) and does the state-of-the-art in "mind-reading" by means of neuroimaging fulfil these standards? Firstly, such a method would have to be able to successfully classify based on low numbers of trials. We call this the signal-tonoise (SNR) challenge. For instance, one could show a particular picture of a crime scene only once to determine whether it is novel or familiar to a suspect. In general, for determining if someone is lying or telling the truth, one could ask "critical questions" or show "critical items" only a few times. In the context of brain-computer/machine interfaces $(\mathrm{BCl}$ or $\mathrm{BMI})$ neuroimaging research has been quite successful in analysing brain signals on a single trial basis with the aim to either control a machine (e.g., wheelchair or a prosthetic) via a computer interface or to use the extracted signals for immediate feedback in clinical rehabilitation (Birbaumer, 2006 and Sorger et al., 2009). However, these findings were obtained with very simple tasks of imagining highly specific movements or visual categories. More sophisticated mind-reading in terms of measuring the content of silent speech of nouns (Mitchell et al., 2008) or visual processing is rapidly developing but is still far away from what is expected of a true mind-reading machine (Logothetis, 2008).

Table 1. Four challenges that future research must address before neuroimaging could be used in court

\begin{tabular}{|c|c|c|}
\hline \multicolumn{2}{|r|}{ Challenge } & Summary \\
\hline 1 & SNR & $\begin{array}{l}\text { Results must be reliable with only a very limited number of trials (single trial analysis in the } \\
\text { worst case). }\end{array}$ \\
\hline 2 & Individuality/norms & $\begin{array}{l}\text { The results must be meaningful and significant for any given individual. Besides dealing with } \\
\text { individual variability issues, this would also require a large number of norms from appropriate } \\
\text { reference populations. }\end{array}$ \\
\hline 3 & Incompliance & $\begin{array}{l}\text { The method must be robust to incompliant participants/suspects. In the research so far, } \\
\text { participants were fully cooperative, even in the few studies with instruction to deceive. }\end{array}$ \\
\hline 4 & Baseline & $\begin{array}{l}\text { Neuroimaging results are always in relation to something'. The baseline of the method must } \\
\text { be reliable even if incompliant participants/suspects try to interfere with the measurement itself. }\end{array}$ \\
\hline
\end{tabular}

Secondly, the method we are looking for would have to generate reliable results for any given individual; i.e., the individuality challenge. Individuals vary in their brain responses, so a large number of norms from the general population is required for locating a given individual within their reference sub-population. Most brain imaging research so far was conducted with small samples and it is unknown how exactly such findings generalise to the whole of the population.

Thirdly, this method would have to be robust in case a participant is incompliant; i.e., the incompliance challenge. So far most research was conducted with volunteers who followed the given instructions. A few studies have begun to investigate deception (Sip et al., 2008), which is an important start. Deception relies on a series of deliberate decisions and processes such as estimation of outcomes, weighting of risk versus reward, monitoring of information exchange to effectively build-up reputation and trust - which essentially 
recruit networks of attention, decision-making and working memory (Sip et al., 2008). All of these cognitive by-products of deception may have typical brain activation patterns that could be used to decode the trustworthiness of statements. However, incompliance imposes an even bigger challenge as participants/suspects might try to interfere with the entire measurement and not simply deceive the experimenter about specific items.

This leads to the baseline challenge: all research in neuroimaging is "in relation to something", i.e., relative to another condition or a neutral period of the trial. Knowing this, suspects could attempt to induce noise into the baseline measurements and thus compromise the analysis of the target trials. As suggested, deception is a complex mental process that recruits networks of attention, decision-making and working memory (Sip et al., 2008). However, knowing this, a suspect could try to over-activate their attention and working memory in relation to all items, incl. the neutral baseline items. For instance, a baseline could be compromised by internally counting backwards from 1000 in steps of two while processing neutral stimuli. Of course, this is pure speculation but it emphasises the kind of research that is needed before neuroimaging could be successfully applied in court.

Although fascinating research is being conducted in the context of 'mind-reading', we can only reach the conclusion that brain imaging is unlikely to be able to contribute at this point in time towards most decisions in court, and that, in fact, it could be dangerous if misused. However, we have suggested a way for establishing an informed knowledge transfer from science into society (Fig. 1) and we have proposed four challenges that brain imaging research would have to address before it could be considered for use in court (Table 1). The issue of whether it should be considered is a related, yet different ethical issue that was beyond the scope of this short communication.

\section{References}

N. Birbaumer

Breaking the silence: Brain-computer interfaces $(\mathrm{BCl})$ for communication and motor control

Psychophysiology, 43 (6) (2006), pp. 517-532

D. Deca, N. Kriegeskorte

Beyond open access: Visions for open evaluation of scientific papers by post-publication peer review

Frontiers in Computational Neuroscience (2010) Call for Special Topic contributions

N. Kriegeskorte, W.K. Simmons, P.S.F. Bellgowan, C.I. Baker

Circular analysis in systems neuroscience: The dangers of double dipping

Nature Neuroscience, 12 (5) (2009), pp. 535-540

\section{T. Kuhn}

The Structure of Scientific Revolutions

University Press, Chicago (1967) 


\title{
N.K. Logothetis
}

What we can do and what we cannot do with fMRI

Nature, 453 (2008), pp. 869-878

T.M. Mitchell, S.V. Shinkareva, A. Carlson, K.M. Chang, V.L. Malave, R.A. Mason et al.

Predicting human brain activity associated with the meanings of nouns

Science, 320 (2008), pp. 1191-1195

\author{
K. Popper \\ Logik der Forschung (The Logic of Scientific Discovery) \\ Springer Verlag, Wien (1934)
}

K.E. Sip, A. Roepstorff, W. Mcgregor, C.D. Frith

Detecting deception: The scope and limits

Trends in Cognitive Sciences, 12 (2) (2008), pp. 48-53

\section{Y.B. Sirotin, A. Das}

Anticipatory haemodynamic signals in sensory cortex not predicted by local neuronal activity Nature, 457 (2009), pp. 475-479

B. Sorger, B. Dahmen, J. Reithler, O. Gosseries, A. Maudoux, S. Laureys et al.

Another kind of 'bold response': Answering multiple-choice questions via online decoded single-trial brain signals

S. Yuval-Greenberg, O. Tomer, A.S. Keren, I. Nelken, L.Y. Deouell

Transient induced gamma-band response in EEG as a manifestation of miniature saccades

Neuron, 58 (3) (2008), pp. 429-441 